\title{
MOLECULAR DYNAMICS SIMULATION OF GRAIN-BOUNDARY DIFFUSION OF VACANCIES IN BCC IRON
}

Thomas Kwok, ${ }^{+}$P.S. Ho, ${ }^{*}$ Sidney Yip, ${ }^{+}$, R.W. Balluffi, ${ }^{++}$

P.D. Bristowe, ${ }^{++}$and A. Brokman ${ }^{++}$

+Department of Nuclear Engineering, Massachusetts Institute of Technology, Cambridge, MA 02139.

${ }^{*}$ T. J. Watson Research Center, IBM Corporation, Yorktown Heights, NY 10598. ${ }^{++}$Department of Materia1s Science and Engineering, Massachusetts Institute of Technology, Cambridge, MA 02139.

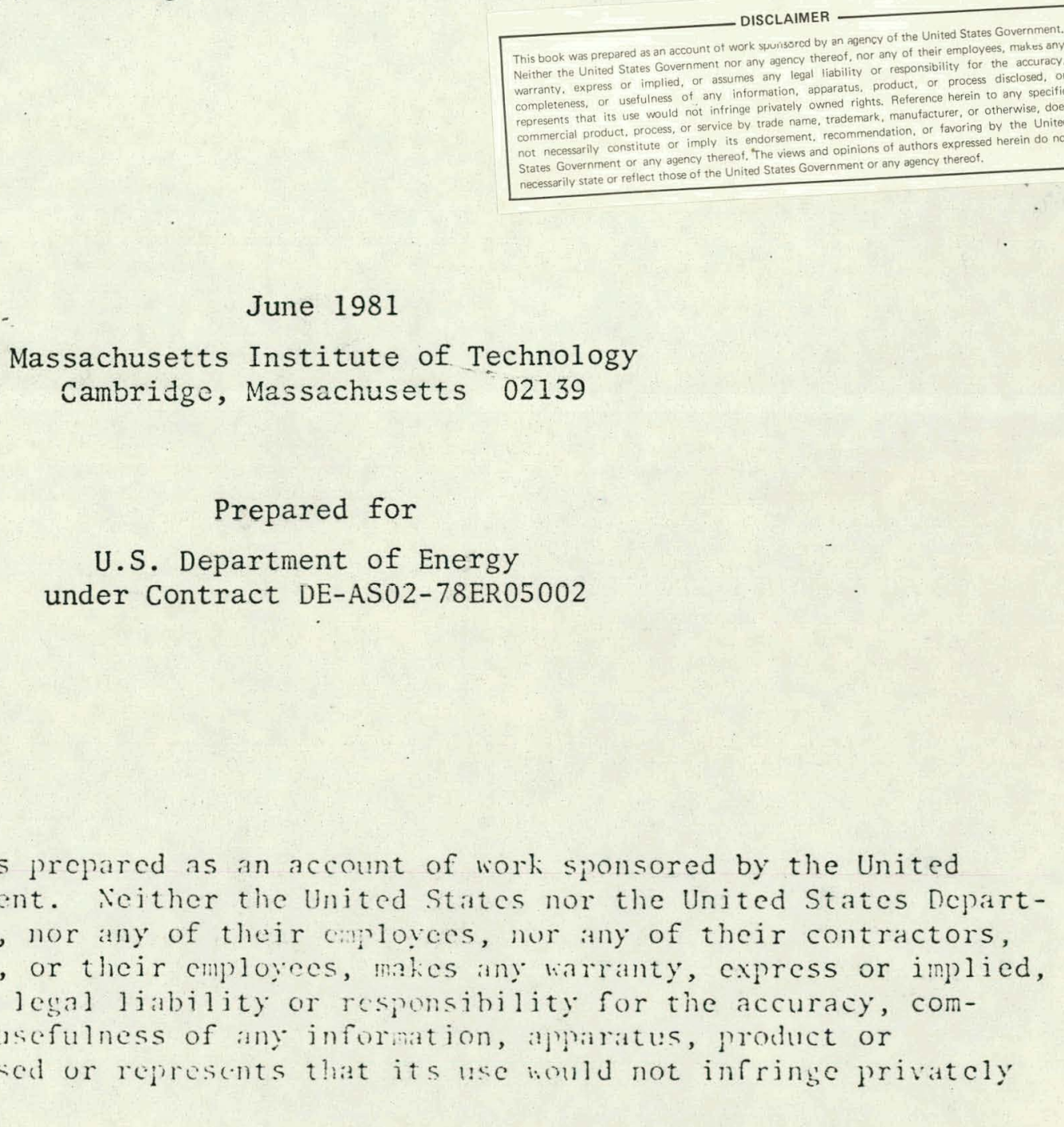

This report was prepared as an account of work siponsored by the United States Goremment. Neither the United States nor the United States Department of Fnersy, nor any of their conloyees, nur any of their contractors, subcontractors, or their employees, makes any warranty, cxpress or implied, or assumes any legal liability or responsibility for the accuracy, completeness, or usefulness of any information, apparatus, product or process disclosed or represents that its use bould not infringe privately owined rights.

t Alsn at Rergman School of Applied Science, Hebrew University of Jerusalem, Israel. 


\section{DISCLAIMER}

This report was prepared as an account of work sponsored by an agency of the United States Government. Neither the United States Government nor any agency Thereof, nor any of their employees, makes any warranty, express or implied, or assumes any legal liability or responsibility for the accuracy, completeness, or usefulness of any information, apparatus, product, or process disclosed, or represents that its use would not infringe privately owned rights. Reference herein to any specific commercial product, process, or service by trade name, trademark, manufacturer, or otherwise does not necessarily constitute or imply its endorsement, recommendation, or favoring by the United States Government or any agency thereof. The views and opinions of authors expressed herein do not necessarily state or reflect those of the United States Government or any agency thereof. 


\section{DISCLAIMER}

Portions of this document may be illegible in electronic image products. Images are produced from the best available original document. 


\title{
Molecular Dynamics Simulation of Grain-Boundary Diffusion of Vacancies in BEC Iron \\ Thomas Kivok, ${ }^{+}$P.S. Ho, " Sidney Yip, ${ }^{+}$R.W. Balluffi, ${ }^{+}$ P.D. Bristowe, ${ }^{++}$and A. Brokman ${ }^{++}:$
}

\begin{abstract}
The jumeing o vacancies in a bcc iron $\Sigma=5$ tilt boundary was simulated by compucer molecular dynamics. The data yielded a reasonable value of the activation energy for migration and showed that the jump processes are righly structure-dependent. The use of a temperature dependent transition probability matrix to describe the diffusion of the vacancies in the grain boundary is suggested. Formation of one type of bounciary interstitial was observed which was found to be immobile.
\end{abstract}

+Department of Nuclear Engineering, MIT, Cambridge; MA 02139

${ }^{*}$ T.J. Watson Research Center, IBM, Yorktown Heights, NY 10598

${ }^{++}$Department of Materials Science and Engineering, HIT, Cambridge, MA 02139 
There has been considerable speculation $(1,2)$ that the fast "short-circuit" self-diffusion which is commonly observed along grain boundaries (GB's) occur by the exchange of atoms with vacancies which are present in the $G B$ in thermal equilibrium. However, it is also conceivable that a defect exchange mechanism involving self-interstitials present in thermal equilibrium may be the dominant mechanism, and it has not yet been possible to demonstrate the predominance of either mechanism because of the difficulty of observing the details of the various jumping processes at the atomic level.

In this letter we present the computer molecular dynamics results of a study of vacancy jumping processes in a bcc iron $\Sigma=5$ $\left(36.9^{\circ}\right)$ [001] (3i0) symmetrical tilt boundary. By exploiting the ability of this technique to simulate at elevated temperatures the detailed atomic motions associated with vacancy jumps, ${ }^{(3-5)}$ we have obtained explicit information on the dependence of these thermally activated processes on the specific GB structure, and how they vary with temperature. Several additional thermally activated processes werc also observed in the $G R$ core during these vacancy diffusion runs which included the spontaneous formation of Frenkel pairs and the interchange of atoms by a completely correlated ring mechanism. Furthermore, the interstitial atom produced in the Frenkel pair formation was found to be completely immobile.

The present results have revealed mobile vacancies and immobile interstitials which lead us to conclude that $G B$ self diffusion occurs by a vacancy exchange mechanism. This point is further discussed and reinforced in Ref. 6. Moreover, the molecular dynamics results suggest that a complete description of the diffusion process be formulated 
in terms of a transition probability matrix which specifies the vacancy jump frequencies between various $G B$ sites.

The model system chosen for study has been investigated recently by the method of molecular statics. (7) The system consisted of a stack of ten layers of atomic planes with their normal along the [001] tilt axis. Each plane contained 40 atomic sites. The equilibrium structure characterized by a free volume in the boundary core corresponding to $82.4 \%$ of an atomic volume $\left(0.34 \mathrm{a}_{0}^{3}\right.$, where $a_{0}$ is the lattice parameter) per period in each layer is shown in Fig. 1(a). All atoms were assumed to interact via an empirical central force, pairwise potential with a Force cutoff midway between second and third neighbors. (8)

The grain boundary was simulated dynamically by applying standard molecular dynamics techniques to the model using periodic border condition in the [001] and [310] directions and fixed borders along the $[730]$ direction. $(9,10)$. Each simulation run was carried out at constant volume;"however, the system volume was adjusted at each temperature to ensure that the system pressure remained essentially atmospheric. A number of isothermal "diffusion runs" were then made in the range $900^{\circ} \mathrm{K}$ to $22100^{\circ} \mathrm{K}$. The $\mathrm{GB}$ structure was found to be stable up to $27650^{\circ} \mathrm{K}$ on the basis of time-average atomic configurations and the local density distribution in the GB region. The vacancy free volume based on a hard sphere model was found to be greater than $60 \%$ of an atomic volume during most of the time between jumps, and consequently, there was no difficulty in locating the vacancy. In the following only resuits at temperatures up to $1550^{\circ} \mathrm{K}$ will be reported. 
During a typical "diffusion run" lasting 22700 lattice vibration periods, some 190 to 360 vacancy jumps were observed depending on the temperature. To define a vacancy jump each atomic site was assigned a spherical volume $\Omega$ equal to the atomic volume at the simulated temperature. An atom undergoing only thermal vibrations therefore would not move out of $\Omega$. A volume $\Omega$ was also assigned to an empty site (i.e., a vacancy), and a successful vacancy jump occurred when an atom was found to move into the $\Omega$ assigned to a vacancy site and remain there for at least two vibration periods.

The major characteristics of the vacancy jumps may be summarized by considering the sites labeled in Fig. $I(a)$ and the data given in Table 1. Each run began with a vacancy at site $B$ in the middle of the system. At the iower temperatures the ensuing jumps were confined mostly to the four sites labeled.A, B, C; and D. (We note that the sites $A^{\prime}, B^{\prime}, \hat{C}^{\prime}$, and $D^{\prime}$ are equivalent sites because of the boundary symmetry.) However, as the temperature was increased jumps to sites further from the boundary midplane became more significant, indicating that they required more thermal activation. This reflects the transition in the grain boundary diffusion kinetics from the so-called "Harrison Type C" regime to the "Harrison Type B" regime. (11). A typical vacancy jlimp path is shown on the right hand side of. Fig. I(a). It is clear from Table 1 that the vacancy migration in the GB shows a strong structure dependence, particularly at the lower temperatures where $G B$ and lattice diffusion rates are significantly different. Another noteworthy feature of our data is that the vacancy trajectories took place predominantly along the tilt axis rather than 
perpendicular to it. This behavior can be understood qualitatively by considering the direction of maximum free volume. There was also significant correlation between successive jumps in contrast to simple random walk events. The distribution of jump directions relative to the preceding jump showed a forward bias similar to that for vacancy jumps in a single lattice. ${ }^{(3)}$

The effective activation energy for $G B$ vacancy jumping, $\hat{E}_{B}^{M}$, was determined by piotting the total vacancy jump frequency (summed over all types of jumps), $\hat{r}$ in the form of an Arrhenius plot in Fig. $T(b)$. A reasonably straight line obeying the relation $\tilde{\Gamma}=\tilde{\Gamma}_{0} \exp \left(-\hat{E}_{B}^{M} / k T\right)$ was obtained with $\mathrm{E}_{B}^{M}=0.51 \mathrm{eV}$ and $\tilde{\Gamma}_{0}=4.85 \times 10^{13} \mathrm{sec}^{-1}$. The pre-exponential factor $\tilde{\Gamma}_{0}^{-}$is seen to be of about the expected magnitude, since, if we write it in the form $\tilde{T}_{0}=\tilde{z}_{0}$, where $\tilde{z}=$ effective coordination number and $\tilde{v}_{0}=$ êtiective "attempt frequency", we obtain $\tilde{v}_{0}=6.06 \mathrm{x}$ $10^{12} \sec ^{-1}$ with $\tilde{Z}=3$. This frequency is of the same magnitude as the Debye frequency, $7.1 \times 10^{12} \mathrm{sec}^{-1}$, corresponding to the Debye temperature of $464^{\circ} \mathrm{K}$ for bcc iron.

The simulation data revealed an interesting interstitial formation at the I locations where there was considerable free volume (cf. Fig. 1(a)). Atoms in B sites were occasionally observed to jump into I sites, the process occurring more frequently as the temperature was increased. This process illustrated in the center of Fig. I(a) may be regarded as the spontaneous formation of a vacancy-interstitial pair, i.c., a Frenkel pair. The vacancy formed in this way often diffused away leaving the interstitial behind at I. Furthermore, the interstitial at I remained completely immobile and could only be eliminated 
by mutual annihilation with a neighboring vacancy. These results indicate that an interstitial in the GB will be strongly trapped at the I sites and therefore will be incapable of promoting self-diffusion.

The dynamical simulations also revealed the interchange of atoms at $B$ and $B^{\prime}$ illustrated in the sequence on the left side of Fig. I(a). In this process an atom in site $B$ jumped into site in the adjacent boundary plane, followed by an atom in site $B^{\prime}$ jumping into the newly created vacancy in the site. $B$. The sequence was then completed by the interstitial in the site I jumping into the vacancy sitting in site $B^{\prime}$. This process also occurred more frequently as the temperature increased; it does not contribute to self-diffusion because the two atoms involved remia in trapped in the same pair configuration, thus producing no net matter transport relative to the other atoms.

At temperatures close to the melting point, a number of infrequent evenis, which were not observed at lower temperatures, were detected. Included were the double jump, which had been observed in previous molecular dynamics studies of vacancy migration in single lattice systems, (4) and jumps over distances greater than the nearest neighbor separation either across the boundary midplane in the same atomic plane of the stack (e.g., jumps between $C$ and $C$ ! in Fig. I(a)) or through the boundary midplane hetween two different atomic planes of the stack.

From the foregoing data it is clear that different boundary sites for vacancy migration are not equivalent, thus suggesting that a proper theoretical treatment of the diffusion of vacancies in the $G B$ as a random walk process requires the use of a set of transition probabilities 
$P_{i j}$ between sites $i$ and $j$. Each GB structure would than be characterized by its transition probability matrix which will not be symmetric and which possesses elements which will vary with temperature.

For purposes of illustration, a transition probability matrix obtained from the $1300^{\circ} \mathrm{K}$ data is shown in Table 2. Here, the matrix has been drastically simplified by including sites $B^{\prime}, C^{\prime} C^{\prime}$ and $D^{\prime}$ in $B, C$ and $D$, so that, for example, jumps between $B$ and $B$ and between $B$ and $B^{\prime}$ are included in the element $P_{B B}$. Also, the elements are normalized so that ${ }_{i}{ }_{j}, P_{B B}=1$. It is interesting to note the inequality $P_{C B}>P_{B C}$ which must be due to a difference between "down-hill" and "up-hill" jumps between sites with different energies. It is worthwhile noting that although the relative magnitudes of the $P_{i j}$ may be estimated approximately from static calculations of vacancy binding energies, molecular dynamics simulation appears to be the only practical means of determining the absolute values of the $P_{i j}$ and their temperature dependences for different sites.

In conclusion, we believe the present results provide the first detalled, microscopic evidence of the migration of vacancies iñ GB's. In Ref. 6 these molecular dynamics data are combined with molecular statics calculations to demonstrate further the vacancy exchange mechanism for $G B$ self diffusion. Further simulations can be expected to yield additional information regarding the characteristics of different $G B$ structures, and the dependence on interatomic potential functions of the diffusion dynamics and structurai stability at various temperatures. 
TK and SY were supported by the U.S.A.R.O., RWB and $A B$ by DOE, and RWB and PDB by NSF. 
References

1. N.L. Peterson, in Grain Boundary Structure and Kinetics, American Society for Metals, Metals Park, Ohio (1980), p. 209.

2. R.W. Balluffi and J.W. Cahn, Acta Met. 29, 493(1981).

3. C.H. Bennett, in Diffusion in Solids, J.J. Burton and A.S. Nowick, eds. (Academic Press, New York, 1975), p. 73.

4. A. Dafano and G. Jacucci, Phys. Rev. Letters 39, 950(1977).

5. An attempt to caiculate the vacancy migration energy in a 6 degree tilt boundary in $\alpha$-iron using a quas $i$-dynamic method of simulation has been made by R.E. Dahl, J.R. Beeler, and R.D. Bourquin, in Interatomic Potentials and Simulation of Lattice DeFects, P.C. Gehlen, J.R. Beeler, R.I. Jaffee, eds. (Plenum Fress, New York, 1972), p. 673.

6. R.W. Bailuizi, Thomas Kwok, P.D.Bristowe, A. Brokman, P.S. Ho, and Sicnay Yip, to be published.

7. A. Brokman, P.D. Brostowe, and R.W. Baliuffi, J. AppT. Phys., in press.

8. R.A. Johnson, Phys. Rev. Al34, 1329(1964).

9. Interatomic Potentials and Simulation of Lattice Defects, P.C. Gehlen, J.R. Beeler, R.I. Jaffee, eds. (PTenum Pres's, New York, 1972).

10. G.H. Bishop, R.J. Harrison, T. Kwok and S. Yip, in Progress in Materials Science, B. Chalmers, H.W. Christian, T.B. Massalski, eds. (Pergamon Press, Oxford), in press.

11. L.G. Harrison, Trans. Faraday Soc. 57, 1191 (1961). 
Table 1. Distribution of observed vacancy jumps into the various sites (see Fig. $7(a)$ ) of a $\Sigma=5$ tilt boundary in bcc iron at three temperatures. (All the unlabeled sites in the model are considered to be $L$ sites. The ratio of the number of each site in the model is $A: B: C: D: E: F: G: L=1: 2: 2: 2: 2: 2: 2: 7$.

\begin{tabular}{rrrrrrrrrr} 
Sites & $A$ & $B$ & $C$ & $D$ & $E$ & $F$ & $G$ & L & A11 \\
\hline $1300^{\circ} \mathrm{K}$ & 3 & 126 & 20 & 32 & 7 & 6 & 1 & 0 & 195 \\
$1400^{\circ} \mathrm{K}$ & 5 & 153 & 34 & 46 & 6 & 9 & 5 & 6 & 264 \\
$1500^{\circ} \mathrm{K}$ & 9 & 197 & 49 & 62 & 11 & 18 & 3 & 4 & 353
\end{tabular}


Table 2 Elements of transition probability matrix $P_{i j}$ for vacancy jump from site $i$ to site $j$ in a $\Sigma=5$ tilt boundary in bcc iron deduced from molecular dynamics simulation data.

\begin{tabular}{l|lllllll}
\multicolumn{1}{c}{$A$} & $B$ & $C$ & $D$ & $E$ & $F$ & $G$ \\
\hline$A$ & .0 & .005 & .010 & .0 & .0 & .0 & .0 \\
$B$ & .0 & .485 & .052 & .108 & .0 & .0 & .0 \\
$C$ & .015 & .072 & .0 & .010 & .005 & .0 & .0 \\
$D$ & .0 & .082 & .031 & .0 & .026 & .026 & .0 \\
$E$ & .0 & .0 & .010 & .021 & .0 & .0 & .005 \\
F & .0 & .0 & .0 & .026 & .005 & .0 & .0 \\
G & .0 & .0 & .0 & .0 & .0 & .005 & .0 \\
.0 & & & & & &
\end{tabular}


Fig. 1 (a) Schematic drawing of the GB model system. Only 3 of the 10 atomic plans and half of the atoms in each plane (which contains 4 coincidence site lattice cells) are shown. The sequence on the right indicates a typical vacancy jump path. The arrow in the center shows an atom at $B$ jumping into the interstitial site $I$. The sequence in the upper left shows the observed interchange of atoms at $B$ and $B^{\prime}$ via a ring mechanism. The ratio of the scale used in the drawing is $[130]:[310]:[001]=1: 1: 5$. (b) Temperature dependence of observed effective vacancy jump irequence $\tilde{I}\left(\mathrm{sec}^{-1}\right)$ in a $\Sigma=5$ tilt boundary in bcc iron. Error bars denote the standard deviation assuming a Poisson distribution. 

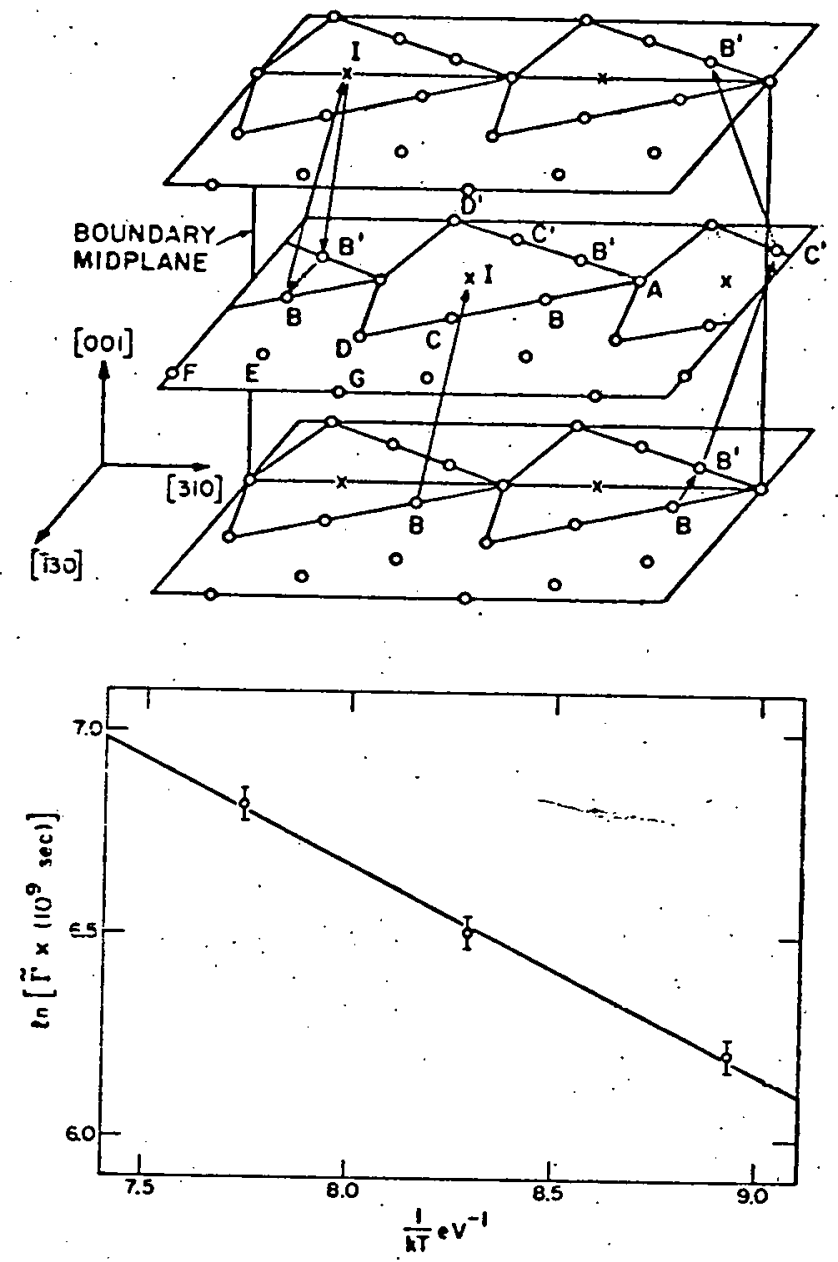